\title{
Neue Entwicklungstendenzen an deutschen Hochschulen
}

\author{
Christoph Helm
}

\section{Einleitung}

Ich freue mich sehr, hier über „Neue Entwicklungstendenzen an deutschen Hochschulen " zu berichten. ${ }^{1}$ Zum einen natürlich, weil aus dem Wunsch nach diesem Referat das fortbestehende Interesse am deutschen Hochschulwesen spricht. Wir wissen um den Wert dieses Interesses und bemuihen uns, es zu erhalten. Zum anderen erhoffe ich mir aus den Diskussionen aber auch weitere Anregungen und Erkenntnisse, um den internationalen Stellenwert deutschen Hochschulen weiter zu steigern.

Erlauben Sie eingangs einen kurzen historischen Exkurs zu den Wurzeln des deutschen Hochschulwesens des früheren 19. Jahrhunderts. Mein Exkurs ist mit dem Namen Humboldt in doppelter Weise verbunden: Ebenso wie Wissenschaft und Forschung mit dem Namen Alexander von Humboldt ist das deutsche Hochschulund Bildungswesen mit dem Namen seines zwei Jahre älteren Bruders Wilhelm von Humboldt verbunden.

\section{Die Hochschulreform Wilhelm v. Humboldts}

Mit der Bestellung Wilhelm v. Humboldts zum Direktor fuir Unterricht und Erziehung im preußischen Innenministerium durch König Friedrich Wilhelm III. im Jahr 1809 erfuhr die Institution Universität eine ihrer umfassendsten Reformen. Mit dem 1810 vorgestellten Gründungskonzept der Universitas litterarum (heute HumboldtUniverstität) als Einheit von Lehre und Forschung, die eine allseitige humanistische Bildung der Studierenden ermöglichen soll, wurde ein neuer Prototyp einer Universität entwickelt, der in den folgenden 150 Jahren weltweit viele Universitäten ihrer Art entstehen ließ.

Was waren die Kernpunkte der Hochschule humboldtscher Prägung?

- Die Einheit von Lehre und Forschung.

- Die Einheit von Lernenden und Lehrenden.

- Die Freiheit der Wissenschaften.

- Die Autonomie der Hochschulen.

- Die Berufung von Professoren durch den Staat und damit die Sicherstellung von Qualität.

- Die Gleichheit aller Fakultäten.

Diese Grundsätze sind auch heute conditiones sine quibus non des deutschen Hochschulwesens. Beibehalten wurde ebenfalls die föderale Struktur des deutschen Bildungswesens, also die Verantwortlichkeit der Bundesländer u. a. für die Hochschulen.

\section{Die Hochschulreform der 90er Jahre}

Allerdings hat sich spätestens zu Beginn der 90er Jahre die Erkenntnis durchgesetzt, dass eine Reform des Hochschulwesens unbedingt notwendig ist, damit die Hochschulen auch künftig ihre bestehenden Aufgaben erfüllen und neuen Herausforderungen gerecht werden können. Ich nenne hier insbesondere die Felder „Neue Rolle der Hochschulen in der Wissensgesellschaft“, „Globalisierung und Internationalisierung“ und „Verknuipfung von Wissenschaft und Wirtschaft“. Insbesondere Letzteres wird zuweilen mit dem Schlagwort „Weg von Humboldt" belegt. Damit wird meiner Meinung nach die Zielrichtung der mittlerweile in Schwung gekommenen Hochschulreform in Deutschland verkannt. Es geht nicht darum, die humboldtschen Prinzipien aufzugeben. Vielmehr müssen sie den neuen Herausforderungen angepasst werden.

Wir befinden uns dabei in einer vergleichbaren Lage wie Wilhelm von Humboldt und seine Zeitgenossen Freiherr vom Stein, Johann Wolfgang von Goethe, Friedrich von Schiller, Johann Gottfried Herder, Immanuel Kant und Jean-Jaques Rousseau. Auch damals stand das deutsche Hochschulwesen einer sich verändernden Welt gegenüber: Bestehende Grenzen - damals zwischen den unzähligen deutschen Kleinstaaten - zerflossen, die Verfügbarkeit von Informationen nahm rasant zu, breite Bevölkerungsschichten mussten Zugang zur Schulbildung erhalten. Die Gesellschaft benötigte besser ausgebildete Akademiker.

\subsection{Rolle der Hochschulen in der Wissensgesellschaft}

Wie sieht es heute aus? Auf dem Arbeitsmarkt wird sich die Nachfrage nach Hochschulabsolventen weiter verstärken. Das Schwergewicht wird sich weiter in Richtung wissenschaftlich fundierter, praxisorientierter Ausbildungs- und Qualifikationsprofile verschieben. Gleichzeitig wird aber auch der Bedarf an Hochschulabsolventen mit forschungsorientierter Ausbildung für Tätigkeiten außerhalb von Lehre und Forschung wachsen. Die Vielfalt der Tätigkeitsbereiche von Hochschulabsolventen und die Dynamik der Veränderung ihres Anforderungsprofils erfordern eine stärkere Differenzierung der Studiengänge und -abschluisse, eine größere Vielfalt der Studienangebote.

\subsection{Neue Abschlüsse/Bolognaprozess}

Der Wissenschaftsrat hat daher im Januar 2000 eine Empfehlung zur Einfuihrung neuer Studienstrukturen 
und -abschlüsse verabschiedet. ${ }^{2}$ Er sprach sich dafür aus, an Universitäten und Fachhochschulen eine klare und flexible Grundstruktur für Studium und Lehre einzuführen.

Das grundständige Studium soll früher als bisher zu einem berufsqualifizierenden Abschluss führen. Neu strukturierte Studienprogramme und neue Studienabschlïsse sollen die unterschiedlichen Interessen Studierender, späterer Arbeitgeber und der Gesellschaft berücksichtigen. Sie sollten generalistische, forschungs- oder anwendungsorientierte Profilschwerpunkte erkennen lassen und das hohe Niveau der wissenschaftlichen Ausbildung in Deutschland wahren. Zur Realisierung des Studienziels „Beschäftigungsfähigkeit“ sind neue inhaltliche und zeitliche Verbindungen zur beruflichen Anwendung und Praxis sowie zu lebenslangem Lernen erforderlich.

Die Einfuihrung neuer Studienstrukturen in Deutschland kann nur eingebettet in den sogenannten Bolognaprozess der EU erfolgen. Im Rahmen des weiteren Ausbaus der EU wird die Schaffung eines einheitlichen Europäischen Hochschulraums angestrebt. In der Bologna-Erklärung vom 19. Juni 1999 haben die für Hochschulwesen zuständigen Minister von 30 europäischen Staaten beschlossen, bis zum Jahr 2010 einen einheitlichen Europäischen Hochschulraum zu verwirklichen und zu diesem Zwecke auf eine Konvergenz der jeweiligen Hochschulsysteme in Europa hinzuarbeiten. Diese Bestrebung haben die Minister in einer turnusmäßigen zweiten Konferenz nach zwei Jahren in Prag bestätigt (Prager Communiqué vom 19.05.2001).

Die nächste Konferenz fand im September 2003 in Berlin statt, auf der eine Berliner Erklärung zu den Fortschritten bzw. bezüglich der Leitlinien und Schwerpunkte fuir die nächsten Schritte des Prozesses zur Schaffung eines Europäischen Hochschulraums veröffentlicht wurde. Die Fassung der Erklärung anlässlich der Berliner Konferenz wird durch die Bologna-Preparatory-Group (BPG) auf europäischer Ebene vorbereitet.

In Umsetzung der Zielstellung der Bologna-Erklärung werden seit Ende der 90er Jahre an deutschen Hochschulen mehr und mehr neue Studiengänge mit den Abschluissen „Bachelor“ und „Master“ angeboten. Mit kürzeren und strafferen Studiengängen ist ein schnelleres Reagieren auf aktuelle Entwicklungen möglich; gleichzeitig können sich die Studierenden entsprechend ihren Interessen und Fähigkeiten für ein Studium entscheiden.

Mit der Einfuihrung von konsekutiven BA/MA-Studiengängen an Universitäten und Fachhochschulen besteht die Notwendigkeit und die Chance,

- das bestehende begrenzte Fächerspektrum der Fachhochschulen deutlich zu erweitern,

- das quantitative Schwergewicht in den Bereich der praxisorientierten Studienangebote, die auf eine wissenschaftlich fundierte Qualifizierung fuir berufliche Tätigkeitsfelder ausgerichtet sind, zu verlagern,

- die Heranbildung eines exzellenten und international konkurrenzfähigen wissenschaftlichen Nachwuchses und die forschungsorientierte Ausbildung für berufliche Tätigkeiten außerhalb von Lehre und Forschung an den Universitäten zu verstärken.

\subsection{Qualitätssicherung}

Mit der Einführung konsekutiver Studiengänge in Deutschland stellt sich die Frage nach einem neuen Verfahren der Qualitätssicherung, das unter Beachtung von Mindeststandards die Vergleichbarkeit und die Qualität von Lehre, Studium und Abschliussen sichert. Akkreditierungsverfahren, wie sie in vielen Ländern bereits Praxis sind, werden als besonders geeigneter Weg angesehen,

- den Wettbewerb zwischen den Hochschulen zu fördern,

- Vielfalt zu ermöglichen,

- Qualität zu sichern und

- Transparenz herzustellen.

Akkreditierungsverfahren müssen, wenn sie die Funktion der Qualitätssicherung erfüllen wollen, überregional angelegt sein. Deshalb wurde in Deutschland im Jahre 2001 ein länderübergreifender Akkreditierungsrat gebildet, dem Wissenschaftler, Vertreter der Berufspraxis, Studierende sowie Vertreter der Hochschulen und der Länder angehören. Die fachlich-inhaltliche Begutachtung der Bachelor- und Masterstudiengänge wird von Akkreditierungsagenturen durchgefuihrt. Gegenstand der Akkreditierung der Studiengänge ist, ob ein bestimmtes Studienangebot von der nach wissenschaftlichen und berufsbezogenen Kriterien zu beurteilenden Qualität und Aktualität geeignet ist, den Studierenden bzw. den Absolventen sinnvolle, berufsverwertbare Kenntnisse zu vermitteln und sich international vergleichbar in ein Gefuige akademischer Studienangebote einzuordnen.

\subsection{Wettbewerb um die besten Köpfe}

Ein weiterer Aspekt der Qualitätssicherung ist die Gewinnung und Auswahl des Lehrpersonals. Es ist ein offenes Geheimnis, dass der „Wettbewerb um die besten Köp$\mathrm{fe}$ " weltweit schärfer geworden ist und dass deutsche Wissenschaftler häufig eine Beschäftigung im Ausland einer Tätigkeit an deutschen Hochschulen vorziehen. In seiner Empfehlung zur Personalstruktur der Universitäten und zur Qualifizierung des wissenschaftlichen Nachwuchses vom Januar 2001 spricht sich der Wissenschaftsrat $^{3}$ für eine Reform der Personalstruktur und der Qualifizierungswege für den wissenschaftlichen Nachwuchs aus. Danach muss die Eignungsfeststellung für Hochschullehrer künftig durch ein im Anspruchsniveau gleichwertiges, international anschlussfähiges und wettbewerbsorientiertes Verfahren erfolgen.

Der Wissenschaftsrat empfiehlt weiter, eine auf fünf Jahre befristete Nachwuchs- oder Juniorprofessur zu schaffen. Nachwuchsprofessoren sind den uibrigen Professoren korporationsrechtlich gleichzustellen und in die Grundausstattung der Fachbereiche einzubinden. Die Besetzung von Nachwuchsprofessuren erfolgt durch ein Berufungsverfahren. Voraussetzungen für die Berufung sind eine herausragende Promotion, Lehrerfahrung und 
zusätzliche wissenschaftliche Leistungen, über deren Umfang die aufnehmende Hochschule je nach Bedarf und Profil entscheiden muss. Der Nachwuchsprofessor muss mindestens einmal die Einrichtung wechseln, entweder bei Berufung oder nach Ablauf der fünf Jahre. Falls er von außen berufen worden ist, sollte die Nachwuchsprofessur nach positivem Ausgang eines Begutachtungsverfahrens nach fuinf Jahren in eine unbefristete Professur umgewandelt werden können (,tenure track“).

Das System der Nachwuchs- bzw. Juniorprofessuren wurde mittlerweile durch die Bundesländer umgesetzt. Wo erforderlich, werden noch in diesem Jahr die Hochschulgesetze angepasst.

\subsection{Stärkere Autonomie der Hochschulen}

Die Hochschulen stehen vor der Herausforderung, sich im internationalen und nationalen Wettbewerb mit anderen Hochschulen erfolgreich behaupten zu müssen. Profilbestimmung/Schwerpunktbildung, Spezialisierung und Kooperation stehen dabei im Mittelpunkt. Mehr Autonomie der Hochschulen ist die Voraussetzung für die Steigerung der Wettbewerbsfähigkeit und die Entfaltung weiterer Leistungspotenziale. Der Staat als wichtigster Förderer der institutionalisierten Wissenschaft muss sich aus der Detailsteuerung der Hochschulen zurïckziehen, der Übergang zu Deregulierung und Dezentralisierung muss konsequent gegangen werden.

Die Leitung einer Hochschule entscheidet uiber die Gestaltung der Fachbereiche und evaluiert sie auf der Grundlage von Lehr- und Forschungsberichten. Sie stellt den Haushalt auf, bewirtschaftet ihn und weist den Fachbereichen Stellen und Mittel befristet und leistungsbezogen zu. Sie bereitet die Konzepte für die Hochschulentwicklung vor und setzt sie nach Entscheidung des Senats um. Gleichzeitig ist sie Dienstvorgesetzter. Dieses Aufgabenprofil erfordert Leiterpersönlichkeiten, die nicht nur in der Scientific Community anerkannt sind, sondern auch Kompetenzen im Managementbereich von Wissenschaft und Wirtschaft haben müssen.

Auf der Fachbereichsebene wurde das Amt des Dekans aufgewertet. Zusätzliche Aufgaben fuihren zu erweiterten Befugnissen. So stellt der Dekan das Lehr- und Prüfungsangebot sicher und erhält in diesem Zusammenhang ein Weisungsrecht gegenuiber den Professoren und Mitarbeitern. Als neue Aufgabe kommt die Evaluation der Einrichtungen des Fachbereichs hinzu, die in Lehr- und Forschungsberichte an den Präsidenten einmünden und zu einer leistungsbezogenen Mittelvergabe fuihren soll.

Um die Mitbestimmung der Mitglieder der Hochschule zu sichern, wird der Senat zum verfassungsgebenden Organ und zugleich unter Zuordnung zusätzlicher Kontrollbefugnisse zum Aufsichtsgremium in akademischen Fragen ausgestaltet.

In Zeiten knapper öffentlicher Finanzen ist eine zielorientierte Steuerung notwendig. Den Hochschulen muss mehr Finanzverantwortung uibertragen werden;
Anreize zu effizienterem wirtschaftlichen Handeln sind zu schaffen. Ein Instrument dafür ist die leistungsbezogene Mittelvergabe. Die leistungsorientierte Mittelverteilung trägt der wachsenden Eigenverantwortung der Hochschulen Rechnung, setzt Anreize für eine Erhöhung der Leistungen auf allen Ebenen und fördert die Wettbewerbsorientierung im Hochschulsystem.

In verschiedenen Ländern werden bereits Modelle zur leistungsbezogenen Mittelvergabe angewendet. Sie beschränken sich in der Regel auf die Zuschüsse für Lehre und Forschung. Alle Modelle differenzieren zwischen einem volumen- bzw. belastungsorientierten und einem leistungs- bzw. anreizorientierten Teil.

Die Anwendung von Modellen der leistungsabhängigen Mittelvergabe ist Teil einer umfassenden Reform der staatlichen Steuerungsmodelle für die Hochschulen. Wesentliche Bausteine des Modells sind: Grundzuweisung, die leistungsbezogene Zuweisung und die Zuweisung für Strukturentwicklung. Die Hochschulen werden Globalhaushalte erhalten, sodass sie die ihnen nach dem Verteilungsmodell zustehenden Mittel flexibel einsetzen und eigenverantwortlich Schwerpunkte setzen können. Ein angemessenes Berichtswesen sorgt dafür, dass der Einsatz der Mittel transparent bleibt und die Hochschulen Rechenschaft über die Verwendung öffentlicher Mittel geben.

An den Hochschulen wird derzeit eine neue Professorenbesoldung eingefuihrt, die leistungsbezogene Elemente beinhaltet und damit auch Anreize für die Erhöhung der Leistungen der einzelnen Professoren gibt.

Zunehmend entstehen private Hochschulen in Deutschland, die das Studienangebot staatlicher Hochschulen zwar nicht ersetzen, aber sinnvoll ergänzen können, innovative Anstöße geben und belebend auf Wettbewerb/Reform des Hochschulsystems wirken.

Die Qualität eines Landes als „Wissenschaftsstandort“ und die Qualität der Hochschulen hängt auch davon ab, ob die zukünftigen Forscher Studienbedingungen vorfinden, die Mit- und Selbsttätigkeit erleichtern. Hochrangige Forschung verlangt auch Nachwuchsförderung. Diese ist aber nur dann wirklich möglich, wenn die Hochschulen der Förderung des wissenschaftlichen Nachwuchses gezielt Priorität einräumen. Die Ressourcen der Hochschulen und der außerhochschulischen Forschungseinrichtungen müssen dafür stärker genutzt, die Kooperation zwischen ihnen muss vertieft werden - das betrifft die Forschungsgegenstände wie die gemeinsame Betreuung des wissenschaftlichen Nachwuchses.

\subsection{Netzwerkbildung/Campus Germany}

Neben der Kompatibilität und Internationalität der Studienabschlüsse ist ein weiteres wichtiges Feld die Vernetzung der Hochschulen. Hier bietet sich übrigens - um zur Familie Humboldt zurückzukehren - Alexander von Humboldt als historisches Vorbild an: Er gilt als einer der größten Kommunikatoren seiner Zeit. Die Namen der- 
jenigen, mit denen er in Kontakt stand, würden Telefonbücher fuillen. Er hat mehr Briefe verfasst als Goethe. Man schätzt deren Zahl auf 50.000 (allein rund 13.500 sind im Wortlaut oder in Ausschnitten erfasst). In unsere Begriffswelt übertragen könnte man ihn durchaus als einen Meister des Networking bezeichnen.

Netzwerkbildung spielt heute fuir die Entwicklung von Lehre und Forschung an deutschen Hochschulen eine wichtige Rolle. Ihre Bedeutung nimmt weiter zu. Dabei beschränkt sich die Vernetzung nicht auf die Hochschulen untereinander. Über den Hochschulsektor hinaus werden mit außeruniversitären Forschungseinrichtungen und der Wirtschaft Verknuipfungen geschaffen. Den Schwerpunkt bilden heute noch regionale Netzwerke zwischen den Hochschulen und Einrichtungen im Bundesland oder der Region. Die uiberregionale und internationale Vernetzung insbesondere in der Lehre steht noch am Anfang. Sie wird aber gezielt vorangetrieben. Ein realistisches, wenn auch fernes Ziel ist die vernetzte europäische Hochschule.

Was bedeutet Vernetzung im Hochschulbereich konkret? ${ }^{4}$ Lassen Sie mich beispielhaft den Prozess der Vernetzung der Hochschulen des Landes Brandenburg darstellen, der - abgesehen von einigen Besonderheiten, die sich insbesondere aus der Lage des Landes am Schnittpunkt zu Osteuropa ergeben - beispielhaft fuir die Entwicklung des deutschen Hochschulwesens gesehen werden kann.

Die Hochschulen des Landes Brandenburg orientieren sich in ihrer Entwicklung am Bild einer offenen und vernetzten Hochschule. Sie reagieren flexibel auf die sich rapide verändernden Bedingungen des Arbeits- und Bildungsmarktes. Sie nehmen die steigenden Legitimationsanforderungen an Lehre und Forschung an. Forschungs- und Entwicklungsvorhaben werden partnerschaftlich mit anderen Hochschulen und Forschungseinrichtungen, der Wirtschaft und der Verwaltungen realisiert. Die Erfahrungen aus diesen Kooperationen wiederum werden für die Entwicklung modularisierter, kombinierter und vernetzter Angebote in der Lehre genutzt. Hier einige Beispiele:

1. An der BTU Cottbus wurde als In-Institut das „Forschungszentrum Bergbaulandschaften“" gegründet. Dieses organisiert sowohl den regional auf das Fallbeispiel Niederlausitz ausgerichteten Forschungsverbund des SFB 565 „Entwicklung und Bewertung gestörter Kulturlandschaften“, als auch das „European Network for long-term Forest Ecosystem and Landscape Research“ ENFORS, das „Research Network Minesite Recultivation“ (RENET) sowie zahlreiche mit weltweiten Partnern durchgefuihrte Forschungsvorhaben. Das Zentrum unterhält ein internationales Forschungsnetzwerk zum Austausch von wissenschaftlichen Ergebnissen und Publikationen, in das aktuell 50 Partnerinstitutionen aus 20 Ländern einbezogen sind. An der Hochschule ist weiter ein „Landeskompetenzzentrum Energie“ im Aufbau, in dem die regionalen Stromerzeuger und -verteiler wie Vattenfall u. a. sowie zahlreiche kleinere Unternehmen in Beziehung zu den energietechnisch und energiewirtschaftlich ausgerichteten Forschungsressourcen der BTU gesetzt werden. Die Landesregierung plant, mit Unterstützung dieses Kompetenzzentrum die „Energiestrategie 2010“ des Landes umzusetzen. Gemeinsam mit der FH Lausitz wird derzeit ein Studiengang „Computational Mechanics“ aufgebaut. Mit der FH Brandenburg wird gemeinsam der Studiengang „,Technologie- und Innovationsmanagement" angeboten.

2. Wissenschaftler der Universität Potsdam arbeiten intensiv mit Forschern der Berliner Hochschulen zusammen. Es entstehen regionale Netzwerke, die äußerst befruchtend auf die Entwicklung der Forschungslandschaft Berlin-Brandenburg wirken. So wurde an der Universität Potsdam ein gemeinsam mit den drei Berliner Universitäten getragener interdisziplinärer Masterstudiengang „Master of Polymer Science“ eingerichtet.

3. Im kürzlich gegründeten „Institutionellen Netzwerk zur Bioinformatik" kooperieren Arbeitsgruppen des Instituts fuir Informatik und des Instituts fuir Biochemie und Biologie der Universität Potsdam, des MaxPlanck-Instituts für Molekulare Pflanzenphysiologie und des Fraunhofer-Instituts für Biomedizinische Technik mit dem Ziel, die Bioinformatik am Wissenschaftsstandort Golm zu fördern und neue Aktivitäten aufzubauen.

4. Die europaweit einmalige wissenschaftliche, mediale und softwaretechnische Kompetenzkonzentration im Raum Potsdam soll fur den Aufbau eines zukunftsorientierten Kompetenzzentrums für nonlineare Medien genutzt werden. Unter Federfuihrung der Hochschule für Film und Fernsehen in Potsdam Babelsberg sollen die medialen nonlinearen Aktivitäten der Universität Potsdam, der FH Potsdam und des Hasso Plattner Instituts zusammengefuihrt werden. Vorgesehen ist eine enge Zusammenarbeit mit Rundfunk- und Fernsehanstalten (u. a. RBB, ARD Digital), mit kleinen und mittleren Unternehmen der Medienindustrie sowie mit weltweit tätigen Großunternehmen.

5. Der Wissenschafts- und Forschungsaustausch zwischen der Europa-Universität Viadrina Frankfurt (Oder) und der Adam-Mickiewicz-Universität Poznan fuihrte zum Aufbau des Collegium Polonicum, einer gemeinsam mit Polen betriebenen Wissenschaftseinrichtung in Slubice, die in der Hochschullandschaft einmalig ist. Ziel ist die Unterstuitzung der Zusammenarbeit polnischer und deutscher Wissenschaftler und Studierender; die Durchfuihrung von Forschungsprogrammen unter besonderer Berücksichtigung der Probleme der europäischen Integration und die vergleichende Forschung unter internationalem und interkulturellem Aspekt.

6. Vom DAAD gefördert wird das International Quality Network „Kopplungsprozesse und ihre Strukturen in der Geo- und Biosphäre“. Es handelt sich um ein Graduiertenzentrum an der Universität Potsdam, das 
den internationalen Austausch von Postdocs und Doktoranden sowie die Mobilität zwischen unterschiedlichen wissenschaftlichen Einrichtungen fördern soll. Beteiligt sind Wissenschaftler aus den Bereichen Erdund Lebenswissenschaften sowie der Mathematik und Physik. Partnereinrichtungen sind im Land Brandenburg ansässige Forschungseinrichtungen.

\subsection{Verknüpfung von Wissenschaft und Wirtschaft/ Stärkung des Praxisbezugs}

\section{a. Fachhochschulen}

Die Fachhochschulen des Landes Brandenburg sind in der anwendungsbezogenen Forschung und Entwicklung äußerst aktiv und werben beachtliche Drittmittel ein. Sie kooperieren dabei insbesondere mit der regionalen Wirtschaft, aber auch mit anderen Hochschulen und außerhochschulischen Forschungseinrichtungen. Die Fachhochschulen haben sich als unverzichtbares Element des deutschen Hochschulsystems erwiesen. Durch anwendungsorientierte Forschung und Entwicklung und die Einrichtung konsekutiver Studiengänge haben sie zusätzliches Profil gewonnen. Allerdings - so forderte der Wissenschaftsrat in seiner Empfehlung zur Entwicklung der Fachhochschulen vom Januar 2002 - muss das vorhandene wissenschaftliche Potenzial künftig noch intensiver genutzt werden. ${ }^{5}$ Der Wissenschaftsrat sieht insbesondere folgende Defizite:

- Der Ausbaustand der Fachhochschulen ist angesichts des zukünftigen Bedarfs an praxisorientiert ausgebildeten Hochschulabsolventen und der inhaltlichen Anforderungen an eine qualitativ hochwertige Ausbildung nicht befriedigend.

- Das Fächerspektrum der Fachhochschulen sollte weiter ausgebaut werden. Die Fachhochschulen können noch nicht in hinreichendem Maße eigenständig Forschung betreiben.

- Wissenschaft und Forschung müssen sich heute ebenso wie die Lehre verstärkt nach dem konkreten Nutzen, sprich dem wirtschaftlichen Wert ihres Wirkens fragen lassen.

\section{b. Duale Studiengänge}

Ein augenfälliges Beispiel für die fließend gewordenen Grenzen zwischen betrieblicher Ausbildung und Hochschulausbildung sind die dualen Studiengänge. Hier wird bereits während des Hochschulstudiums begleitend eine betriebliche Ausbildung absolviert. Das duale System zielt auf eine größere Praxisnähe und ganzheitliches Denken in technischen und wirtschaftlichen Zusammenhängen. An der Fachhochschule Wildau werden gegenwärtig in enger Kooperation mit Unternehmen zwei duale Studiengänge mit einer Regelstudienzeit von drei Jahren erprobt: Bioinformatik/Biosystemtechnik und Telematik. Die Praxispartner stammen überwiegend aus der Region Berlin-Brandenburg.

Anfang 2001 startete das Bundesministerium für Bildung und Forschung eine Initiative zur Bündelung optischer Technologien in regionalen Netzwerken. Ziel ist es, die optischen Technologien zu einer wesentlichen Querschnitt-Schliusseltechnologie zu entwickeln. Eines von insgesamt acht Kompetenznetzen für „Optische Technologien“ in Deutschland ist OpTecBB e.V. In Zusammenarbeit zwischen den Technischen Fachhochschulen in Berlin und Wildau sowie der Fachhochschule Brandenburg und Wirtschaftsunternehmen aus dem Raum BerlinBrandenburg wurde der Modellstudiengang „Master of Engineering in Photonics“ geschaffen. Kooperationsverträge und Zielvereinbarungen fixieren die von allen Seiten zu erbringenden Leistungen.

\section{Schlussbetrachtung}

Die mit den Namen Wilhelm v. Humboldts verbundene Universitätsidee zu Beginn des 19. Jahrhunderts hat das Hochschulwesen in Brandenburg/Preußen und Deutschland nachhaltig befruchtet. Die durch sie bewirkte Entwicklung der Wissenschaft, insbesondere der Naturwissenschaften, hat die preußischen und deutschen Universitäten in der Zeit des Kaiserreiches in eine fuihrende Position gebracht.

Wenn gegenwärtig so viel Bewegung in die deutschen Hochschulen gekommen ist, hat das damit zu tun, dass die Hochschulen den veränderten Rahmenbedingungen nicht mehr gerecht werden konnten. Das Humboldtsche Prinzip der Einheit von Lehre und Forschung ließ sich unter den Bedingungen der Massenuniversität, wie sie sich vielerorts in Deutschland herausgebildet hat, nicht mehr für alle Studierenden verwirklichen. Hinzu kam, dass die Praxis nicht von allen Hochschulabsolventen eine gleichermaßen hohe Forschungsbefähigung erwartet. Nur an diejenigen, die Forschungs- und Entwicklungsaufgaben zu lösen haben, sind entsprechende Erwartungen gerichtet.

Das neue konsekutive Ausbildungssystem wird den differenzierten Praxisanforderungen an die Hochschulabsolventen gerecht. Es gewährleistet, dass die MasterAbsolventen eine dem Prinzip der Einheit von Lehre und Forschung entsprechende Forschungsqualifizierung erfahren. So gesehen geht es bei den sich gegenwärtig vollziehenden Veränderungen im deutschen Hochschulwesen um die Bewahrung des Humboldtschen Erbes in Deutschland. Am Ende dieser Entwicklung, die mit Differenzierungen zwischen den Hochschulen verbunden ist, gibt es dann hoffentlich herausragende Fakultäten und herausragende Hochschulen, die den Vergleich mit internationalen Spitzenuniversitäten nicht zu scheuen brauchen und für Studieninteressierte aus aller Welt attraktiv sind.

\section{Anmerkungen}

1 Zu den Neuansätzen ab 1990, die sich aus dem Umbau des Wissenschaftssystems in den neuen Bundesländern ergeben haben, vgl. Christoph Helm/Eberhard Ambos/ Dierk Behrmann/Hannes König: Erfahrungen und Erkenntnisse zur Gewinnung von Studenten für das Ingenieurstudium, in: Gießerei 86, Zeitschrift der Deutschen Gießereivereinigungen, 12/1999, S. 65-68. 
ders.: Verwaltungsinformatik als Teil der Hochschulentwicklung und Hochschulreform in Sachsen-Anhalt, in: Verwaltungsinformatik 2000. 3. Internationale Fachtagung „Verwaltungsinformatik“ der Gesellschaft für Informatik, S. 181-188, Halle 2000.

ders.: Ludwig Ross und seine Bedeutung fuir die Klassischen Altertumswissenschaften, Akzidenzen 12, Flugblätter der Winckelmann-Gesellschaft, Stendal 2000. (auch abgedruckt in: Orientwissenschaftliche Hefte I/2001, Orientwissenschaftliches Zentrum der Martin-Luther-Universität Halle-Wittenberg, S. 1-13).

ders.: Zehn Jahre Wissenschaftsentwicklung in den neuen Bundesländern an Hand des Paradigmas Sachsen-Anhalt, Kurzinformation HIS, Hannover 2001.

ders.: Hochschulen und Forschungseinrichtungen Ostdeutschlands in Kooperation mit Osteuropa, in: Forum der Forschung. Wissenschaftsmagazin der Brandenburgischen Technischen Universität Cottbus. Heft 12, S. 13-33, Cottbus 2001.

ders.: Europäische Integration, Bildung und Forschung die Erfahrungen des Landes Brandenburg. in: Societas Humboldtiana Polonorum, Band 9, S. 117-125, Warschau 2001.

ders.: Innovation für Sachsen-Anhalt, in: Aufbruch. Festschrift zur zehnjährigen Gründung des Standortes Dessau der Hochschule Anhalt, S. 15-19, Dessau 2002.

ders.: Stärkung der Wettbewerbsfähigkeit durch den Aufbau regionaler Innovationssysteme, in: Regionale Innovationssysteme, Wildauer Schriftenreihe Innovationsmanagement, Bd. 2, S. 19-24, Wildau 2002.

ders.: Ausblick: Neues Lernen und Multimedia, in: Wissenschaftliche Beiträge der Fachhochschule Lausitz, 1. Ausgabe, S. 69-74, Senftenberg/Cottbus 2002.

ders., Die Fachhochschulen in den Hochschulsystemen der Länder. Möglichkeiten und Grenzen in Brandenburg, in: Hochschullehrerbund, Band 43, Heft 5, S. $40-44$, Bonn 2002.

2 Vgl. Empfehlung zur Einfuihrung neuer Studienstrukturen und -abschliusse (Bakkalaureus/Bachelor - Magister/Master) in Deutschland vom 21.01.2000 (Drs. 4418/00).

3 Vgl. Personalstruktur und Qualifizierung: Empfehlungen zur Förderung des wissenschaftlichen Nachwuchses vom 19.01.2001 (Drs. 4756/01).

4 Vgl. dazu im Detail:

Helm: Wissenschaftsentwicklung, S. $25 \mathrm{ff}$.

ders.: Hochschulen, S. $18 \mathrm{ff}$.

ders.: Integration, S. $120 \mathrm{ff}$.

ders.: Wettbewerbsfähigkeit, S. $22 \mathrm{ff}$.

5 Vgl. Empfehlungen zur Entwicklung der Fachhochschulen vom 18.01.2002 (Drs. 5102/02).

\section{Autor}

\section{Dr. Christoph Helm}

Staatsstekretär im Ministerium für Wissenschaft, Forschung und Kultur des Landes Brandenburg

Dortustraße 36, 14467 Potsdam

Tel. +49 3375 866-4557

E-Mail: christoph.helm@mwfk.brandenburg.de 